\title{
THE STABILITY OF HARROD'S GROWTH MODEL OF AN ECONOMY
}

\author{
P. E. LUSH \\ (received 22 March 1964, revised 10 December 1964)
}

\section{Introduction}

Nevile [2] has shown that if $R_{t}$ is a certain measure of the rate of growth of the national income in Harrod's growth model of an economy, then $R_{t}$ satisfies the non-linear recurrence relation

$$
k R_{t+2} R_{t+1}-R_{t+1}^{2}+R_{t}+c R_{t+1}=0, \quad t=0,1,2, \cdots,
$$

where $0<k<1$ and $-1<c<1$. The definition of $R_{t}([2]$ p. 369) is such that $R_{t}>0$ for all $t$. Nevile has pointed out features of the model that indicate that it may be unstable. In this paper I propose to show that the model is, in general, unstable, but that proper choice of the initial values $R_{\mathbf{0}}, R_{\mathbf{1}}$ apparently leads to stability. In order to do this, we require the conditions (if any) under which $R_{t}$ converges.

By writing (1) as

$$
R_{t}-R_{t+1}=R_{t+1}\left\{R_{t+1}-k R_{t+2}-(c+1)\right\},
$$

it follows that the only possible limits for $\left\{R_{t}\right\}$ are zero and $(c+1) /(1-k)$. In the economic setting the latter limit is the one of practical interest, and I propose to concentrate attention upon this case.

Chaundy and Phillips [1] have discussed the sequence that is defined by a certain two-term quadratic recurrence relation - their example shows that the behaviour of such a sequence is dependent on the initial value (as well as the parameters) being suitably restricted. I will show that a similar property holds for the recurrence relation (1), in particular, the sequence so defined converges if and only if the initial point $\left(R_{1}, R_{0}\right)$ lies on a curve in the $R_{1}-R_{0}$ plane. In such cases the convergence is monotonic. (Cf. [6].)

If we describe (1) by $S_{t}=\phi\left(S_{t+2}, S_{t+1}\right)$, where the function $\phi$ is subjected to the conditions (4), (5), (9) and Lemma 5 (below), then the conclusion of the previous paragraph remains valid for this more general case; and, as a by-product, it follows that there exists a continuous, monotonic solution of the functional equation 


$$
f(f(x))=\phi(f(x), x)
$$

Similar results have been obtained in [3], [4], [5] and [7] for this functional equation under conditions upon the function $\phi$ that are essentially different to those employed in this paper. The condition imposed in Lemma $\mathbf{5}$ is not "natural", but I have not been able to deduce it from the other conditions, nor have I been able to avoid its use.

\section{Preliminaries}

By writing equation (1) as

$$
R_{t+2} / R_{t+1}=k^{-1}\left\{1-\left(c+R_{t} / R_{t+1}\right) R_{t+1}^{-1}\right\}
$$

we see that

(i) if $R_{t}<R_{t+1}$ and $R_{t+1}>(c+1) /(1-k)$, then $R_{t+1}<R_{t+2}$;

(ii) if $R_{t}>R_{t+1}$ and $R_{t+1}<(c+1) /(1-k)$, then $R_{t+1}>R_{t+2}$.

An equality in either of the premises of (i) or (ii) would not affect the conclusions. If $R_{0}<R_{1}$ and $R_{1}>(c+1) /(1-k)$, the sequence is monotonically increasing, and, as each term exceeds the greatest possible limit, the sequence diverges to infinity. If $R_{0}>R_{1}$ and $R_{1}<(c+1) /(1-k)$, the sequence decreases monotonically away from $(c+1) /(1-k)$. If we suppose that the sequence approaches the only possible limit, viz. $R_{t} \rightarrow 0$, then for $R_{t} \geqq R_{t+1}$ and for $R_{t+1}$ sufficiently small, it follows from (3) that $R_{t+2}$ is negative, contradicting the assumption that $R_{t}$ is positive for all $t$. Statements (i) and (ii) imply that $\left\{R_{t}\right\}$ cannot oscillate about $(c+1) /(1-k)$, and there remain for consideration the cases of monotonic convergence.

For definiteness consider the case of monotonic decrease to the limit. If we put $S_{t}=R_{t}(1-k) /(c+1)$, then (1) has the form $S_{t}=\phi\left(S_{t+1}, S_{t+2}\right)$, where

the function $\phi(x, y)$ is of class $C_{3}$ and

(i) $\phi(1,1)=1, \phi(x, x)>x$ if $x>1, \phi(x, x)<x$ if $x<1$;

(ii) $\phi_{y}<0$ for $x>0, y>0$.

(Note that if $S_{t+1}>1$, then by (4) (i),

$$
\phi\left(S_{t+1}, S_{t+1}\right)>S_{t+1},
$$

and if $S_{t+1}>S_{t}$ then

$$
\phi\left(S_{t+1}, S_{t+1}\right)>\phi\left(S_{t+1}, S_{t+2}\right),
$$

so that $S_{t+2}>S_{t+1}$ by (4) (ii). Likewise, if $S_{t}>S_{t+1}$ and $S_{t+1}<1$, then 
$S_{t+2}<S_{t+1}$, and thus the sequence $\left\{S_{t}\right\}$ cannot oscillate about the limit.) Assume that, in addition to $(4), \phi(x, y)$ satisfies

(i) $\phi_{x}+\phi_{y}>1$ for $1 \leqq y \leqq x$;

(ii) $\phi_{x y}+\lambda \phi_{y y}<0$ for $x \geqq 1, y \geqq 1,0 \leqq \lambda \leqq 1$.

It follows from (4) (ii) that there is region $H$ formed by the union of $(1,1)$ with the points $(x, y)$ for which

$$
\phi(x, x)<y<\phi(x, 1), x>1
$$

and the sequence $\left\{S_{t}\right\}$ converges monotonically to one if and only if, for $t=0,1,2, \cdots$, the point $\left(S_{t+1}, S_{t}\right)$ belongs to $H$. Let $T$ be the transformation such that $T[(x, y)]=(\alpha, \beta)$ where

$$
\alpha=y, \beta=\phi(y, x) \text {. }
$$

If $(x, y)$ is $\left(S_{t+2}, S_{t+1}\right),(\alpha, \beta)$ is $\left(S_{t+1}, S_{t}\right)$ and $\left\{S_{t}\right\}$ converges if and only if $\left(S_{t+1}, S_{t}\right)$ belongs to $T[H]$ for $t=0,1,2, \cdots$.

\section{Some Lemmas}

Lemma 1. If we write $T\left[T^{j}[H]\right]=T^{j+1}[H]$, then

$$
H \supset T[H] \supset T^{2}[H] \supset \cdots \text {. }
$$

Proof. Call $y=G_{0}(x)$ and $y=g_{0}(x)$ the upper and lower boundaries of $H$ respectively *. From (5) (i) $g_{0}^{\prime}(x)>1$, so that $g_{0}(x)$ has a single-valued inverse, $g_{0}^{-1}(x)$ (say), satisfying $1<g_{0}^{-1}(x)<x$ for $x>1$, and $g_{0}^{-1}(1)=1$. Let $T\left[x, g_{0}(x)\right]=g_{1}(x)$, then

$$
\begin{aligned}
g_{1}(x) & =\phi\left(x, g_{0}^{-1}(x)\right), \\
& >\phi(x, x)=g_{0}(x),
\end{aligned}
$$

by (4) (ii). Similarly, if $T\left[x, G_{0}(x)\right]=G_{1}(x)$,

$$
g_{0}(x)<g_{1}(x)<G_{1}(x)<G_{0}(x), \quad x>1,
$$

with $g_{1}(1)=G_{1}(1)=1$. It follows from (4)(ii) that an interior point of $H$ transforms into a point $(\alpha, \beta)$ satisfying $g_{1}(\alpha)<\beta<G_{1}(\alpha)$; thus $H \supset T[H]$.

Since $g_{0}^{\prime}(x)>1$,

$$
\begin{gathered}
g_{1}^{\prime}(x)=\phi_{x}+\phi_{y} / g_{0}^{\prime}(y), y=g_{0}^{-1}(x), \\
>\phi_{x}+\phi_{y},
\end{gathered}
$$

by (4)(ii); and it then follows from (5)(ii) that

* viz. $\mathrm{G}_{0}(x)=\phi(x, 1)$ and $g_{0}(x)=\phi(x, x)$. 


$$
\begin{aligned}
g_{\mathrm{I}}^{\prime}(x) & >\phi_{x}+\phi_{y}, y=x, \\
& =g_{0}^{\prime}(x)>1 .
\end{aligned}
$$

This argument can be rephrased to show formally by induction that $H \supset T[H] \supset T^{2}[H] \supset \cdots$, and the sequence $\left\{S_{t}\right\}$ will then converge if and only if $\left(S_{1}, S_{0}\right)$ is restricted to the limit set $L$ of this nest of sets.

Call $y=G(x), y=g(x), \quad(G(1)=g(1)=1)$, the upper and lower boundaries of $L$ respectively. I propose to show that $G(x)=g(x)$ for $x>1$ - to do this let us suppose that $G(x)>g(x)$ for $x>1$. ${ }^{*}$ Call $y=G_{n}(x)$, $y=g_{n}(x)$ the upper and lower boundaries of $T^{n}[H]$ respectively, and let the Taylor expansion of $\phi(x, y)$ about $(1,1)$ be $1+a(x-1)-b(y-1)+\cdots$, where, from (5)(i), $a-b>1$.

LEMMA 2. The sequence $\left\{g_{n}^{\prime}(1)\right\}$ increases monotonically to

$$
p=\frac{1}{2}\left\{a+\left(a^{2}-4 b\right)^{\frac{1}{2}}\right\}
$$

whilst $\left\{G_{n}^{\prime}(1)\right\}$ decreases monotonically to the same limit.

Proof. The last paragraph in the proof of Lemma 1 shows that $G_{n}^{\prime}(x)>1$ for $x \geqq 1$, so that we can write

$$
G_{n+1}(x)=\phi(x, y), y=G_{n}^{-1}(x),
$$

giving

$$
G_{n+1}^{\prime}(1)=a-b / G_{n}^{\prime}(1) .
$$

From (4) and (5) $a>0, b>0$, and it follows by induction that $G_{n+1}^{\prime}(1)<G_{n}^{\prime}(1)$; likewise, (i) $\left\{g_{n}^{\prime}(1)\right\}$ is monotonically increasing, and (ii) $G_{n}^{\prime}(1)>g_{n}^{\prime}(1)$. Thus the two sequences are convergent - the possible limits being the roots of the equation

$$
p^{2}-a p+b=0 \text {. }
$$

Since $a-b>1$ there are two roots of this equation - the larger root satisfies $G_{0}^{\prime}(1)>p>g_{0}^{\prime}(1)$, whilst the smaller root, being less than $g_{0}^{\prime}(1)$, does not provide a possible limit. Thus both $\left\{g_{n}^{\prime}(1)\right\}$ and $\left\{G_{n}^{\prime}(1)\right\}$ converge to the expression (6).

The smaller root of $(8)$ is

$$
\frac{1}{2}\left[(a-2)-\left\{(a-2)^{2}+4(a-b-1)\right\}^{\frac{1}{2}}\right]+1,
$$

and as $a-b-1>0$, we then have the

COROLLARY. The larger root of (8) is greater than $b$.

* If we suppose the other alternative, viz. $g(x)=G(x)$ for $1 \leqq x \leqq x^{*}$ and $g(x)<G(x)$ for $x^{*}<x$, the argument then moves to the second last paragraph of $\S 4$. 
LEMma 3. Let $Q$ be the triangle $1 \leqq y \leqq x \leqq \xi_{1}$, and suppose that, for all $(x, y)$ in $Q$, there is a constant $K_{1}$ such that

$$
K_{1}>\phi_{x x y}+2 \phi_{x y} \lambda+\phi_{y y} \lambda^{2}>0,0 \leqq \lambda \leqq 1 ;
$$

then $G_{n}^{\prime \prime}(x)>0, g_{n}^{\prime \prime}(x)>0$. If we put $\alpha=\phi_{x x x}(1,1), \beta=\phi_{x y}(1,1), \gamma=\phi_{y y}(1,1)$, then there are fixed numbers $\delta, K_{2}$ such that for $1 \leqq x \leqq 1+\delta$

$$
G_{n}^{\prime \prime}(x)<\alpha /\left(1-b p^{-3}\right)+2 \beta \lambda_{n-1}+\gamma \lambda_{n-1}^{2}+K_{2}(x-1),
$$

where $\lambda_{n}=1 / G_{n}^{\prime}(1)$

Proof. From (7) there follows

$$
G_{n+1}^{\prime \prime}(x)=\phi_{x x}+2 \phi_{x v} \lambda+\phi_{y y} \lambda^{2}-\phi_{y} G_{n}^{\prime \prime}(y) \lambda^{3}, \quad \lambda=1 / G_{n}^{\prime}(y) .
$$

The last paragraph of the proof of Lemma 1 shows that $0 \leqq \lambda \leqq 1$, and as $G_{0}^{\prime \prime}(x)=\phi_{x x}$, it follows by induction from (9) and (4) (ii) that $G_{n}^{\prime \prime}(x)>0$; similarly $g_{n}^{\prime \prime}(x)>0$. Let $K=\max \left(\left|\phi_{x x}\right|,\left|\phi_{x y}\right|,\left|\phi_{y y}\right|\right)$ for all $(x, y)$ in $Q$, then

$$
\left|\phi_{y}\right|<b+2 K(x-1)
$$

as $G_{n}^{-1}(x)<x$.

As $b<p$, we choose a fixed number $\delta>0$, less than $\frac{1}{2}(p-b) / K$; then, whenever $1 \leqq x \leqq 1+\delta$,

$$
\left|\phi_{y}\right| / p<(b+2 K \delta) / p<1 .
$$

Now $G_{n}^{\prime \prime}(x)>0$, so that $\lambda \leqq 1 / p$, and it follows from (10) and (11) that $G_{n}^{\prime \prime}(x)$ is uniformly bounded in $1 \leqq x \leqq 1+\delta$; similarly $g_{n}^{\prime \prime}(x)$ is uniformly bounded. Now $G_{0}^{\prime \prime}(x)=\phi_{x w}$, and as $\phi$ is of class $C_{3}$,

$$
G_{1}^{\prime \prime}(x)<\alpha\left(1+b p^{-3}\right)+2 \beta \lambda_{0}+\gamma \lambda_{0}^{2}+K_{3}(x-1),
$$

where $K_{3}$ is some constant. Since $\lambda$ and $G_{n}^{\prime \prime}(x)$ are uniformly bounded in $1 \leqq x \leqq 1+\delta$, (10) gives

$$
G_{n+1}^{\prime \prime}(x)<\alpha+2 \beta \lambda_{n}+\gamma \lambda_{n}^{2}+b G_{n}^{\prime \prime}(y) \lambda_{n}^{3}+K_{4}(x-1),
$$

where the constant $K_{4}$ is independent of $n$. As $y<x$ and $\lambda_{n}<1 / p$, the Lemma follows by use of (5)(ii) if we choose $K_{2}=\left\{\max \left(K_{3}, K_{4}\right)\right\} /\left(1-b / p^{3}\right)$.

LEMMA 4. For a chosen $\xi_{1}, G_{n}^{\prime}(x), g_{n}^{\prime}(x)$ are uniformly bounded, and $G(x), g(x)$ are continuous in $1 \leqq x \leqq \xi_{1}$.

Proof. It follows from (7) and (4)(ii) that

$$
G_{n}^{\prime}(x)<\phi_{x}(x, y), y=G_{n}^{-1}(x)>1,
$$


and so by (5) (ii) $G_{n}^{\prime}(x)<\phi_{x}(x, 1)$. But $G_{n}^{\prime}(x)>1$ and thus the sequence $\left\{G_{n}(x)\right\}$ is equicontinuous in $1 \leqq x \leqq \xi_{1}$; so that, as it converges monotonically, it converges uniformly. $G_{n}(x)$ is continuous, and so $G(x)$ is continuous; similarly for $g(x)$.

LEMMA 5. Let

$$
A=-(\beta+\gamma / p)-b\left\{\alpha /\left(1-b / p^{3}\right)+2 \beta / p+\gamma / p^{2}\right\} / p^{2},
$$

then there is a fixed number $x^{*}>0$ such that, for $1 \leqq x \leqq x^{*}$ and $n \geqq N(\varepsilon)$, $G_{n}^{\prime}(x)-g_{n}^{\prime}(x)>0$ if $A>0$.

Proof. If we put $y=g_{n}^{-1}(x)$ and $Y=G_{n}^{-1}(x)$, use of (7) leads to

$$
G_{n+1}^{\prime}(x)-g_{n+1}^{\prime}(x)=A(x)(y-Y)-\phi_{\nu}(x, y)\left\{G_{n}^{\prime}(y)-g_{n}^{\prime}(y)\right\} /\left\{G_{n}^{\prime}(Y) g_{n}^{\prime}(y)\right\},
$$

with

$$
A(x)=G_{n}^{\prime \prime}(\bar{x}) \phi_{y}(x, y) /\left\{G_{n}^{\prime}(Y) g_{n}^{\prime}(y)\right\}-\left\{\phi_{x y}\left(x, y_{1}\right)+\phi_{y y}\left(x, y_{2}\right) / G_{n}^{\prime}(Y)\right\},
$$

where $\bar{x}, y_{1}, y_{2}$ are some numbers in $(Y, y)$. As $\phi$ is of class $C_{3}$ and as $\beta<0$, $\gamma<0$, we use Lemma 3 and $G_{n}^{\prime}(1)>p>g_{n}^{\prime}(1)$ to give

$$
A(x)>A_{1}-K(x-1), 1 \leqq x \leqq 1+\delta,
$$

where $K>0$ is a constant independent of $n$ and

$$
\begin{aligned}
A_{1}= & -\beta\left[1+2 b /\left\{p^{2} G_{n-1}^{\prime}(1)\right\}\right]-\gamma\left[1 / G_{n}^{\prime}(1)+b\left\{p G_{n-1}^{\prime}(1)\right\}^{-2}\right] \\
& -b \alpha /\left\{p\left(1-b / p^{3}\right) g_{n}^{\prime}(1)\right\} .
\end{aligned}
$$

For any prescribed $\varepsilon$ we may, by Lemma 2 , choose an $N(\varepsilon)$ such that

$$
p-\varepsilon<g_{n}^{\prime}(1)<G_{n}^{\prime}(1)<G_{n-1}^{\prime}(1)<p+\varepsilon ;
$$

thus

$$
A_{1}>A-K_{1} \varepsilon
$$

where $K_{1}>0$ is independent of $\varepsilon$.

However $G_{N}^{\prime}(1)>g_{N}^{\prime}(1)$, and, by Lemma 3 , there is a fixed number $\delta_{1}>0$ such that $G_{N}^{\prime}(x)-g_{N}^{\prime}(x)>0$ for $1 \leqq x \leqq 1+\delta_{1}$. If $A>0$ we choose $\varepsilon<A /\left(2 K_{1}\right)$ and $x^{*}=1+\min \left(\delta, \delta_{1}, A / 2 K\right)$, then $A(x)>0$ and the Lemma follows by induction.

\section{The Main Theorem}

THEOREM. If $\phi(x, y)$ satisfies the conditions (4), (5), (9) and * $A>0$, there exists a curve $L: y=f(x), \xi_{0} \leqq x \leqq \xi_{1}$, such that the sequence generated

* $A$ is defined in lemma 5 with $p=\frac{1}{2}\left\{a+\left(a^{2}-4 b\right)^{\frac{1}{2}}\right\}, a=\phi_{x}(1,1), b=\phi_{y}(1,1)$, $\alpha=\phi_{x x}(1,1), \beta=\phi_{x y}(1,1), \gamma=\phi_{v y}(1,1)$. 
by $S_{t}=\phi\left(S_{t+2}, S_{t+1}\right)$ converges to 1 if and only if the initial terms $\left(S_{1}, S_{0}\right)$ lie on $L$. The function $f(x)$ provides, at the same time, a continuous and strictly increasing solution of the functional equation

$$
f(f(x))=\phi(f(x), x),
$$

such that (i) $f(1)=1$, and (ii) $f(x)>0$.

Proof. I propose to show that $\lim \left\{G_{n}(x)-g_{n}(x)\right\}=0$. Let the line $y=x_{0}$ intersect the curves $y=G_{n-1}(x), y=g_{n-1}(x)$ at $x_{1}, x_{2}$ respectively. Then $x_{1}<x_{2}<x_{0}$ and

$$
\begin{aligned}
x_{2}-x_{1} & =g_{n-1}^{-1}\left(x_{0}\right)-G_{n-1}^{-1}\left(x_{0}\right), \\
& =\left\{G_{n-1}\left(x_{2}\right)-g_{n-1}\left(x_{2}\right)\right\} / G_{n-1}^{\prime}(\theta),
\end{aligned}
$$

for some number $\theta$ in $x_{1}<\theta<x_{2}$. From Lemmas 2 and 3, $G_{n-1}^{\prime}(\theta)>p$, and as $x_{2}<x_{0}$

$$
g_{n-1}^{-1}\left(x_{0}\right)-G_{n-1}^{-1}\left(x_{0}\right)<\left\{G_{n-1}\left(x_{0}\right)-g_{n-1}\left(x_{0}\right)\right\} / p,
$$

providing $x_{0} \leqq x^{*}$ and $n \geqq N(\varepsilon)$. Using (7) and (12) we have

$$
\left\{G_{n}\left(x_{0}\right)-g_{n}\left(x_{0}\right)\right\} /\left\{G_{n-1}\left(x_{0}\right)-g_{n-1}\left(x_{0}\right)\right\}<-\phi_{y}\left(x_{0}, \eta\right) / p,
$$

for some number $\eta$ in $G_{n-1}^{-1}\left(x_{0}\right)<\eta<g_{n-1}^{-1}\left(x_{0}\right)$; and it then follows from (11) that $\left\{G_{n}\left(x_{0}\right)-g_{n}\left(x_{0}\right)\right\} \rightarrow 0$, uniformly for $1 \leqq x_{0} \leqq x^{*}$. As $G_{n}(x), g_{n}(x)$ are strictly increasing, the limit set $L$ defined in the last paragraph of the proof of Lemma 1 is a curve i.e. $G(x)=g(x)$ for $1 \leqq x \leqq x^{*}$.

Now $G(x), g(x)$ possess inverses and satisfy the functional equation

$$
f(x)=\phi\left(x, f^{-1}(x)\right),
$$

whence, by (4) (ii), $G(x)=g(x)$ if and only if $G^{-1}(x)=g^{-1}(x)$. Let $P$ be the point on $L$ for which $x=x^{*}$, and let $P^{\prime}$ be the reflection of $P$ in $y=x$. Since $g(x)>p(x-1)+1$, it follows that, at $P^{\prime}, x>1+p\left(x^{*}-1\right)$, i.e. $g^{-1}(x)=G^{-1}(x)$ for $1 \leqq x \leqq 1+p\left(x^{*}-1\right)$, and the set $L$ is then a curve for $1 \leqq x \leqq 1+p\left(x^{*}-1\right)$. By repetition of the argument, it follows that $L$ is a curve for $1 \leqq x \leqq \xi_{1}$.

The region $0<x \leqq 1$ corresponds to monotonic increase to the limit, and a similar argument holds for this case. It is necessary to restrict $x$ so that $g(x)>0$, and for this it is sufficient to require $x \geqq \xi_{0}$, where $\xi_{0}$ is the largest root of $\phi(x, 1)=0$ in $[0,1]$. Lemma 4 then shows that, in $\xi_{0} \leqq x \leqq \xi_{1}$, there exists a strictly increasing continuous solution of the functional equation

$$
f(x)=\phi\left(x, f^{-1}(x)\right)
$$


or, what is equivalent, $f(f(x))=\phi(f(x), x)$, such that (i) $f(1)=1$, and (ii) $f(x)>0$.

\section{Conclusion}

For the difference equation (1)

$$
\phi(x, y)=(c+1)\left(x^{2}-k x y\right) /(1-k)-c x ;
$$

so that (4), (5), and (9) are satisfied with $\xi_{1}=\infty$. The condition $A>0$ of Lemma 5 is satisfied for all points $(k, c)$ of the rectangle $-1<c<1$, $0<k<1$ which are exterior to the closed curve $A=0$ shown in Fig. 1.

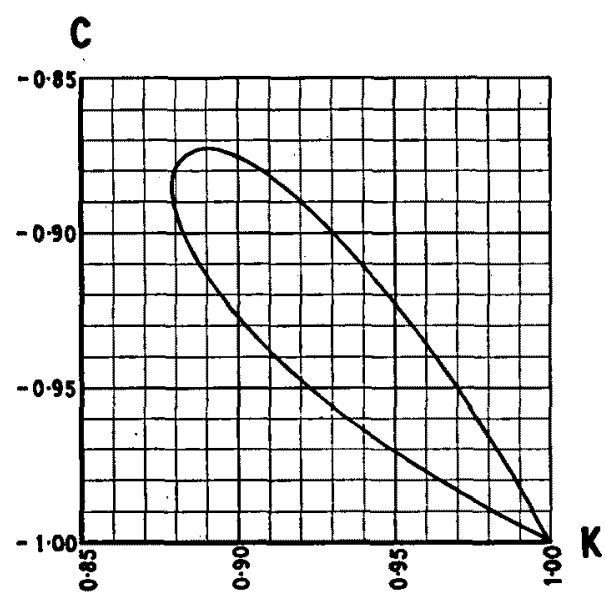

Fig. 1 .

As the points in the interior of the curve $A=0$ are not realised in practice, the significance of the result is that the model of an economy represented by (1) is unstable, in the sense that, with the inaccuracies that are inherent in the measurement of economic quantities, the probability that any given "initial" point $\left(R_{1}, R_{0}\right)$ should lie on $L$ is zero.

\section{References}

[1] Chaundy, T. W. and Phillips, E., The convergence of sequences defined by quadratic recurrence-formulae, Quart. J. of Math. (Oxford) 7 (1936), 74-80.

[2] Nevile, J. W., The mathematical formulation of Harrod's growth model, Econ. J. 72 (1962), 367-370.

[3] Kuczma, M., On monotonic solutions of a functional equation I, Ann. Polon. Math. 9 (1960), 295-297.

[4] Kuczma, M., On monotonic solutions of a functional equation II, Ann. Polon. Math. 10 (1961), $161-166$. 
[5] Fort, M. K., Continuous solutions of a functional equation, Ann. Polon. Math. 13 (1963), $205-211$.

[6] Panov, A. M., The behaviour of the solutions of difference equations near a fixed point, Izv. Vyss. Ucebn. Zaved. Matematika (1959), no. 5 (19). 174-183. (Russian).

[7] Choczewski, B., On continuous solutions of some functional equations of the n-th order, Ann. Polon. Math. 11 (1961), 123-132.

University of New England, Armidale. 